\title{
IVUS-based imaging modalities for tissue characterization: similarities and differences
}

\author{
Hector M. Garcìa-Garcìa • Bill D. Gogas • \\ Patrick W. Serruys • Nico Bruining
}

Received: 8 December 2010/Accepted: 30 December 2010/Published online: 17 February 2011

(C) The Author(s) 2011. This article is published with open access at Springerlink.com

\begin{abstract}
Gray-scale intravascular ultrasound (IVUS) is the modality that has been established as the golden standard for in vivo imaging of the vessel wall of the coronary arteries. The use of IVUS in clinical practice is an important diagnostic tool used for quantitative assessment of coronary artery disease. This has made IVUS the de-facto invasive imaging method to evaluate new interventional therapies such as new stent designs and for atherosclerosis progression-regression studies. However, the gray-scale representation of the coronary vessel wall and plaque morphology in combination with the limited resolution of the current IVUS catheters makes it difficult, if not impossible, to identify qualitatively (e.g. visually) the plaque morphology similar as that of histopathology, the golden standard to characterize and quantify coronary plaque tissue components. Meanwhile, this limitation has been partially overcome by new innovative IVUS-based post-processing methods such as: virtual histology IVUS (VH-IVUS, Volcano Therapeutics, Rancho Cordova, CA, USA), iMAP-IVUS (Bostoc Scientific, Santa Clara, CA, USA), Integrated Backscatter IVUS
\end{abstract}

H. M. Garcìa-Garcìa · B. D. Gogas .

P. W. Serruys · N. Bruining $(\square)$

Department of Interventional Cardiology, Thoraxcenter, Erasmus University Medical Center, Room BA-571, Dr.

Molewaterplein 40, 3015, GD, Rotterdam, The

Netherlands

e-mail: n.bruining@erasmusmc.nl
(IB-IVUS) and Automated Differential Echogenicity (ADE).

Keywords Intravascular ultrasound . Radiofrequency data analysis - Atherosclerosis · Tissue characterization

\section{Introduction}

Atherogenesis is the leading cause of cardiovascular mortality and morbidity in the developed world. The imaging of coronary atherosclerosis and more in particular the high risk atheromatous plaque has made an explosive progress the last decade with the advent of innovative techniques that focus on the high-resolution visualization of the coronary vascular wall. Gray-scale intravascular ultrasound (IVUS) is the modality that has been established as the golden standard for in vivo imaging of the vessel wall of the coronary arteries [1,2]. The use of IVUS is an important diagnostic tool used for quantitative assessment of coronary artery disease. This has made IVUS the de-facto invasive imaging method of choice to evaluate new interventional therapies such as new stent designs [3-5] and for atherosclerosis progression-regression studies [6-14]. However, the grayscale representation of the coronary vessel wall and plaque morphology in combination with the limited 
resolution of the current IVUS catheters makes it difficult, if not impossible, to identify qualitatively (e.g. visually) the plaque morphology similar as that of histopathology, the golden standard to characterize and quantify coronary plaque tissue components. Meanwhile, this limitation has been partially overcome by new innovative IVUS-based post-processing methods such as: virtual histology IVUS [15, 16] (VH-IVUS, Volcano Therapeutics, Rancho Cordova, CA, USA), iMAP-IVUS [17] (Bostoc Scientific, Santa Clara, CA, USA), Integrated Backscatter IVUS [18] (IB-IVUS) and Automated Differential Echogenicity [19] (ADE).

\section{Intravascular ultrasound (IVUS) principles}

The size of current IVUS catheters are ranging from 2.6 to 3.5 French $(0.87-1.17 \mathrm{~mm})$ and are inserted into the coronary arteries through 6-French guiding catheters. The principle of IVUS imaging is based on the oscillatory movement (expansion and contraction) of a piezoelectric transducer (crystal) in order to produce sound waves when electrically excited. There are two major different transducer designs [2]: (1) the mechanical single element rotating device and (2) the electronic phased array. The mechanical rotating element device uses a single piezoelectric transducer that rotates with 1,800 rotations per minute, while the electronic phased array device uses multiple stationary placed piezoelectric transducers which are sequentially activated. The generated sound waves by the transducers propagates through the different tissues and is reflected according to the acoustic properties of the tissue it travels through [20].

Gray-scale IVUS based atheromatous plaque classification is limited due to its low spatial resolution and for the usual IVUS transducers (20 and $40 \mathrm{MHz}$ ) for which the axial resolution is $200 \mu \mathrm{m}$ and the lateral 200-250 $\mu \mathrm{m}$. Based on their visual appeareance, not necessarily histological composition, atheromas have been classified in four categories by gray-scale IVUS: (1) soft plaque (lesion echogenicity less than the surrounding adventitia), (2) fibrous plaque (intermediate echogenicity between soft (echolucent) atheromas and highly echogenic calcified plaques), (3) calcified plaque (echogenicity higher than the adventitia with acoustic shadowing), and (4) mixed plaques (no single acoustical subtype represents $>80 \%$ of the plaques) [2].

\section{IVUS based imaging modalities for tissue characterization}

To overcome the limitations of qualitative visual interpretation of the IVUS images and to describe the coronary plaque morphology, several post-processing methods for computer-assisted quantification have been developed during the recent years. There are two basic different approaches: (1) Signal-based analysis (the so called raw radiofrequency analysis or RF-analysis) and (2) Image-based analysis. The different methods will be described below:

\section{Tissue characterization using virtual histology IVUS (VH-IVUS)}

The first commercial available RF-signal based tissue composition analysis tool was the so-called virtual histology (VH-IVUS, Volcano Therapeutics) software. It uses in-depth analysis of the backscattered RF-signal in order to provide a more detailed description of the atheromatous plaque composition and is performed with either a $20 \mathrm{MHz}, 2.9 \mathrm{~F}$ phasedarray transducer catheter (Eagle Eye ${ }^{\mathrm{TM}}$ Gold, Volcano Therapeutics) or $45 \mathrm{MHz}$ 3.2F rotational catheter (Revolution ${ }^{\circledR}$, Volcano Therapeutics) that acquires IVUS data electrocardiogram gated [21]. The main principle of this technique is that it uses not only the envelope amplitude of the reflected RFsignals (as gray-scale IVUS does), but uses also the underlying frequency content to analyze the tissue components present in coronary plaques. This combined information is processed using autoregressive models and thereafter in a classification tree that determines four basic plaque tissue components [15]: (1) Fibrous tissue (dark green), (2) Fibrofatty tissue (light green), (3) Necrotic core (red) and (4) dense calcium (white). The current software version assumes the presence of a media layer, which is artificially added, positioned just at the inside of the outer vessel contour. This technique has been compared in several studies against histology in humas and other species, see Table 1 for further details. 


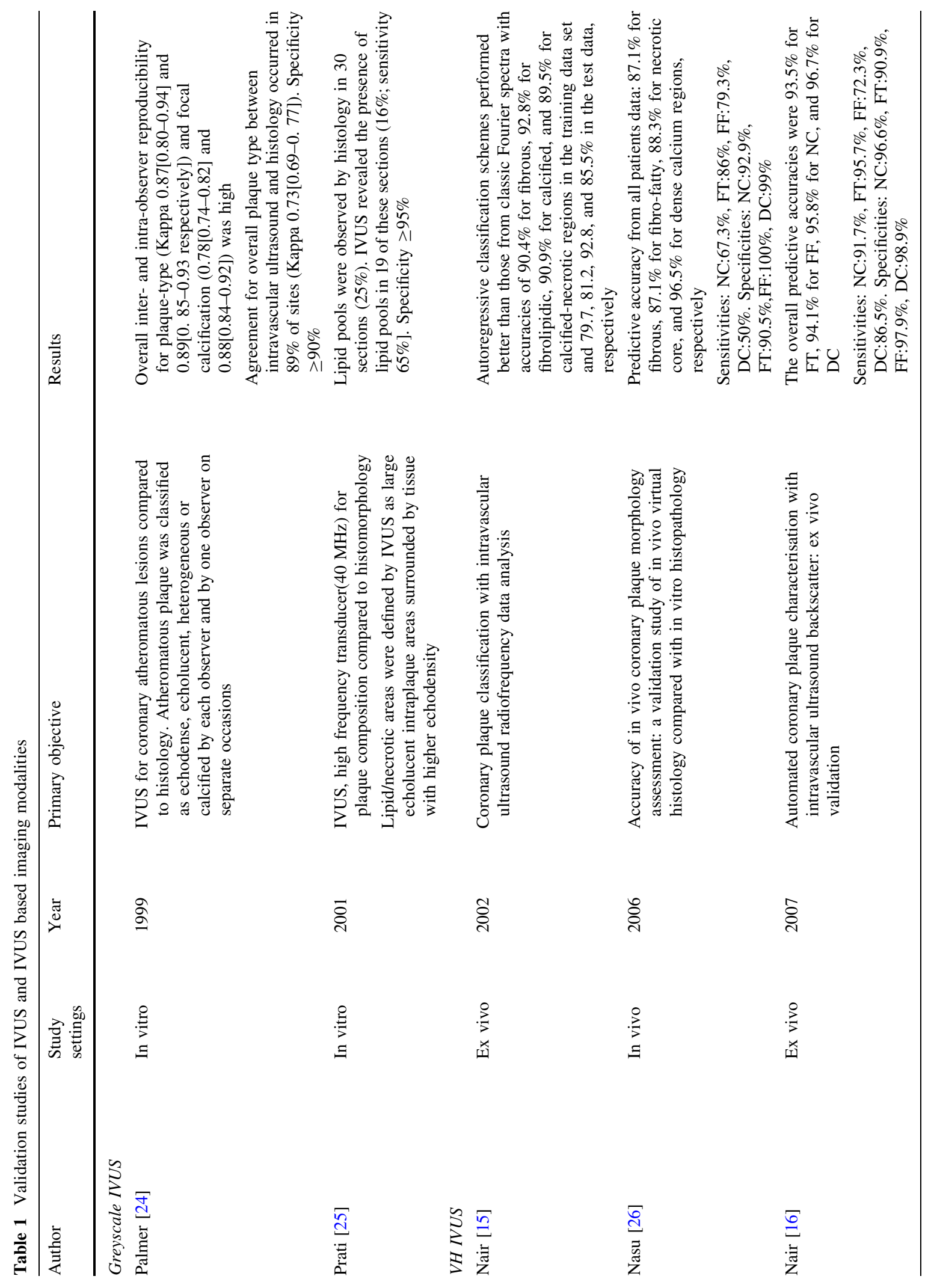




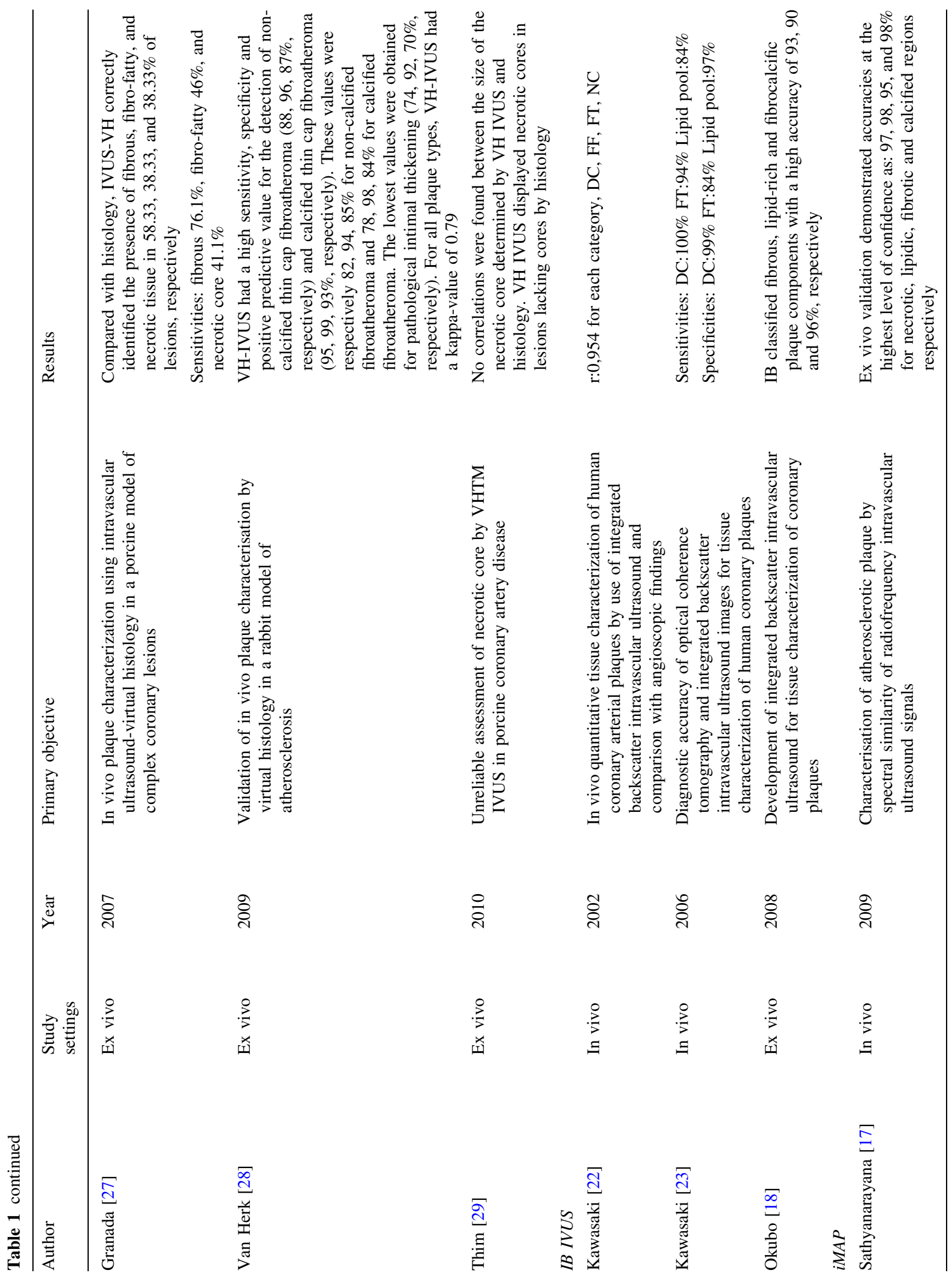




\section{Tissue characterization using i-MAP-IVUS}

Recently, another RF-based processing method has become commercially available for coronary plaque tissue characterization which is called iMAP-IVUS (Boston Scientific) [17]. In principle this software is comparable, from methodological point of view, in brief IVUS-RF parameters are processed using autoregressive models and matched to a database of known RF-signal profiles containing the characteristics of four tissue types. The colour-coded images contain the different colors overlaying the gray-scale IVUS images. However, there are differences, to start with the applied colour scheme: (1) Fibrous tissue (light green), (2) Lipid tissue (yellow), (3) Necrotic core (pink) and (4) Calcium (blue) [17]. Furthermore, the applied IVUS catheter is a $40 \mathrm{MHz}$ rotating single element catheter. Ex vivo validation demonstrated accuracies at the highest level of confidence as: $97 \%$, $98 \%, 95 \%$, and $98 \%$ for necrotic, lipidic, fibrotic and calcified regions respectively (Table 2) [17].

\section{Tissue characterization by integrated backscatter IVUS analysis (IB-IVUS)}

IB-IVUS analysis is an alternative approach, as compared to the 2 previous ones, using the RF-signals of the IVUS catheters to characterize coronary plaque tissue components. IB-IVUS analyses the RF-signals generated by the $40 \mathrm{MHz}$ mechanically rotating IVUS catheters by applying a fast Fourier transformation of the frequency components of the backscattered signals calculating the intensity of the signal measured in decibels (dB). Different tissue components reflect the RF-signals at different power levels, which, according to the developers, could be used to differentiate various tissue components. Analogue to the other methods it also applies a colour coded overlay onto the gray-scale IVUS images. It comprises the following different tissue components: (1) Calcification, (2) Fibrous tissue, and (3) Lipidic [18, 22, 23].

\section{Tissue characterization using, automated differential echogenicity (ADE)}

Automated differential echogenicity is a post-processing imaging analysis method that uses IVUS 
Table 2 Similarities and differences of IVUS and IVUS-based imaging modalities

\begin{tabular}{|c|c|c|c|c|c|}
\hline & Gray-scale IVUS & VH & i-MAP & $\begin{array}{l}\text { Integrated } \\
\text { backscatter }\end{array}$ & Echogenicity \\
\hline Type of device & $\begin{array}{l}\text { Mecanical and } \\
\text { electrical }\end{array}$ & $\begin{array}{l}\text { Mechanical and } \\
\text { electrical }\end{array}$ & Mechanical & Mechanical & As IVUS \\
\hline Transducer frequency & $20-40 \mathrm{MHz}$ & $20-45 \mathrm{MHz}$ & $40 \mathrm{MHz}$ & $40 \mathrm{MHz}$ & As IVUS \\
\hline Colour code & Gray-scale & $\begin{array}{l}\text { Fibrous: green } \\
\text { Necrotic core: red } \\
\text { Callcium: white } \\
\text { Fibrofatty: light } \\
\text { green }\end{array}$ & $\begin{array}{l}\text { Fibrous: light green } \\
\text { Necrotic core: pink } \\
\text { Calcium: blue } \\
\text { Fibrofatty: yellow }\end{array}$ & $\begin{array}{l}\text { Fibrous: green } \\
\text { Necrotic core: blue } \\
\text { Calcium: red } \\
\text { Fibrofatty: yellow }\end{array}$ & Gray-scale \\
\hline $\begin{array}{l}\text { Backscatter radiofrequency } \\
\text { signal analysis }\end{array}$ & Amplitude (dB) & $\begin{array}{l}\text { Autoregressive } \\
\text { model }\end{array}$ & $\begin{array}{l}\text { Fast Fourier } \\
\text { trannformation }\end{array}$ & $\begin{array}{l}\text { Fast Fourier } \\
\text { transformation }\end{array}$ & $\begin{array}{c}\text { Computer } \\
\text { analysis }\end{array}$ \\
\hline
\end{tabular}

images to characterize atheromatous coronary plaque components [19]. At variance with the previous described RF-signal analysis methods, it makes use of the differences in gray-value intensities within the gray-scale IVUS images which are caused by the different acoustic properties (e.g. echogenicity) of the different tissue components. It is known that adventitia tissue contains mostly fibrotic tissue components. Using the relative gray-value of the adventitia layer surrounding the external elastic membrane (EEM), which within quantitative IVUS analysis is detected and defined as the outer vessel wall, tissue components can be differentiated as hypo- (lower gray-values than the adventitia) or hyperechogenic (higher gray-values than the adventitia). Bright structures (very high gray-levels) combined with acoustic shadowing can be identified as calcium. The method classifies structures within these acoustic shadowed areas as unknown as the ultrasound signal is mostly completely reflected by thick layers of calcium and is not able to penetrate it (Figs. 1 and 2). The method has not been developed, such as the other methods, to generate colour-coded IVUS images that try to mimic as close as possible images as generated by histopathology, but to quantify differences over time to evaluate possible changes of the plaque components induced by new therapeutic treatments. It is of outmost importance, in order to better define the images, to follow a certain algorithm that includes: (1) accurate plaque identification (e.g. quantitative IVUS), (2) tunica adventitia definition and grey-level intensity determination (3) normal distribution of the gray-level intensity histogram of the adventitia tissue (image quality control), (4) acoustic shadowing and calcification identification [19]. The advantages of this method are that it can be performed retrospectively on already acquired IVUS data and it is independent of the applied IVUS systems and/or catheters. However, it is recommended to apply within a longitudinal study the same console type and catheter during baseline and follow-up examinations not only within this method but also with respect to the other methods.

\section{Discussion}

As above described, IVUS has become over the past 20 years an important clinical intracoronary imaging tool. It not only facilitates clinical practice but it is also a reference method of which its quantitative parameters are often used as endpoints in first-in-man studies and larger clinical trials. However, the limited resolution, as compared to histology, and gray-scale (256 shades of gray are used when the image is optimal) representation, for which we humans have only limited capabilities with our eyes to make a distinction between them (on average we can only discriminate 8-12 different gray-levels) combined with the large amount of images acquired during a pullback examination (in an IVUS study of $4 \mathrm{~cm}$ there are 2,400 individual cross-sections acquired), requires another representation of the information we are looking for. Of course quantification is mandatory for research purposes and thus automated or semiautomated computer-assisted methods are necessary. In addition, humans can interpret colour-information much better than gray-scale information alone and 


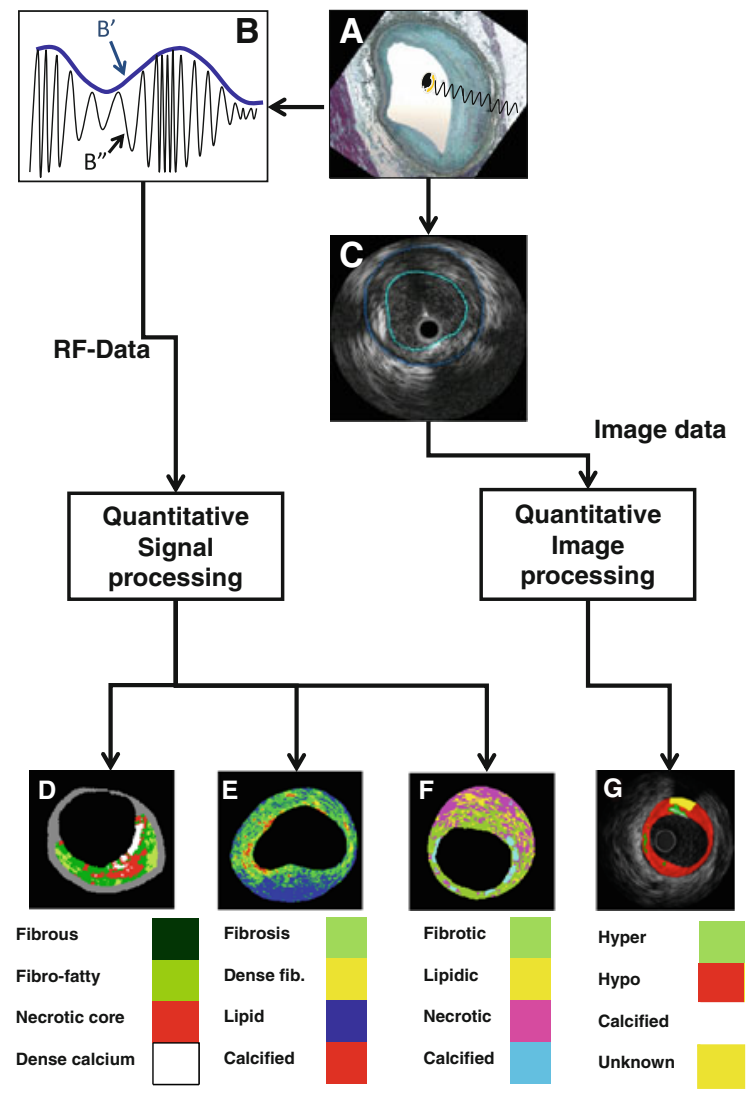

Fig. 1 A shows how an intravascular ultrasound signal is obtained from the vessel wall within an histology image. The greyscale IVUS image, as can be appreciated in $\mathbf{C}$, is formed by the envelope (amplitude) ( $\left.\mathrm{B}^{\prime}\right)$ of the radiofrequency signal, which is illustrated in $\mathbf{B}$. By greyscale atherosclerotic plaque can be classified into 3 categories: hypoechogenic, hyperechogenic and calcified. A 4th category is defined as unknown, which is tissue that is acoustically showed $(\mathbf{G})$. C shows a cross-sectional view of a grayscale image. The blue lines limit the actual atheroma. The frequency and power of the signal commonly differ between tissues, regardless of similarities in the amplitude. From the backscatter radiofrequency data (identified by $\mathrm{B}^{\prime \prime}$ in $\mathbf{B}$ ) different types of tissue information can be retrieved: virtual histology (D), integrated backscattered (IB) IVUS (E) and iMAP (F). Virtual histology is able to detect four tissue types: necrotic core, fibrous, fibro-fatty and dense calcium. The tissues characterized by integrated backscattered (IB) IVUS are lipidic, fibrous and calcified; and iMAP detects fibrotic, lipidic, necrotic and calcified

therefore the plaque compositional tools as described in this overview are valuable additions to the use of standard IVUS gray-scale imaging alone. Until recently, there was only one tool (e.g. VH IVUS) commercially and thus widespreadly available. This has changed, now more methods are available which could potentially lead to different outcomes as they are using different mathematical methods and software algorithms. Certainly, in the near future the different methods will possibly be applied side-byside in multi-center studies and it is yet unknown if this could result in possible different study outcomes, this needs to be explored in future research.

The name giving of virtual histology IVUS (VHIVUS) could possibly lead to expectations that the results derived by this method are one-to-one comparable to histology, which is unfortunately not the case. The development of the RF-signal based methods have been performed empirically, in brief: explanted vessels are imaged by IVUS, the pathologist performs histology and the histology results are cross-correlated to the IVUS images. The derived and correlated RF-signal patterns have been used to build the signal pattern databases which are later used to identify the plaque tissue components in vivo. The crux in this development process is the crosscorrelation between histology and IVUS, which due to the large differences in image resolution is difficult (by example the lateral resolution of IVUS is $200-250 \mu \mathrm{m}$ while that for histology is $5 \mu \mathrm{m}$ ). Although histology is considered the golden standard it is also not free from possible artifacts, by example during the fixation and staining process. Interpretation of the images by different pathologists could also result in interobserver related-biases. It is therefore not realistic to expect similar results from the IVUS derived tissue compositional tools as from quantitative histology. However, if appropriately used, and with justified expectations, these quantitative plaque tissue compositional tools, with all of their limitations, can be of great additional value to investigate plaque compositional changes which cannot be performed with any other imaging method. Great care must thus be taken when trying to prove a hypothesis using these methods which could be out of range of the capabilities of the methods and the basic method, e.g. IVUS itself. An example of expectations which could lead to disappointing results when the RF-based methods are applied is within metallic stented segments. The RF-based methods do not have the signal profile of metallic stent struts in their tissue signal profile databases and this will thus result in an 


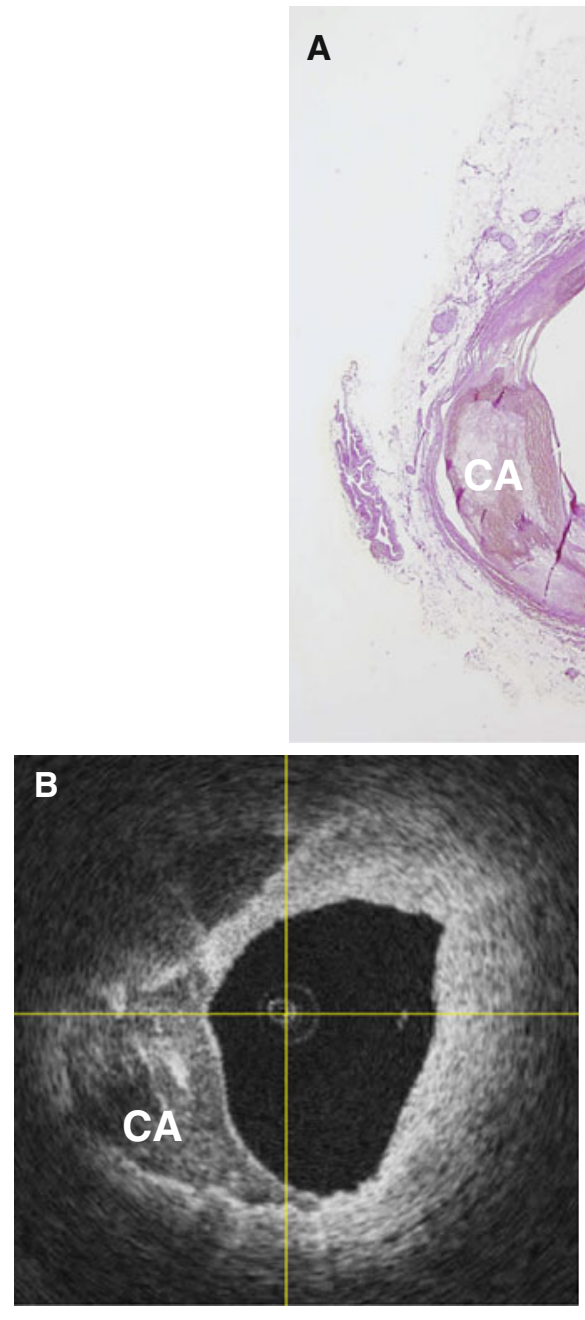

Fig. 2 One of the main limitations of processing the RAW radiofrequency data only, not taking into account other information available, such as visible acoustic shadowing, is that these signal processing methods identify tissue components in these acoustic shadowed regions while IVUS does not return any information about it. The ultrasound waves are reflected at the calcium interface as can be appreciated in this figure. A presents a histology sample of an explanted human coronary artery of which in B the OCT image is presented and in $\mathbf{C}$ the IVUS equivalent. It can be appreciated that OCT

artificial identification (in some research papers this is identified as misuse). This could later lead to unpredictable results and thus care must be taken applying these methods for research for which they are not designed.

However, encouraging results have been achieved applying the tissue composition methods to detect and to quantify as a surrogate the absorption of the

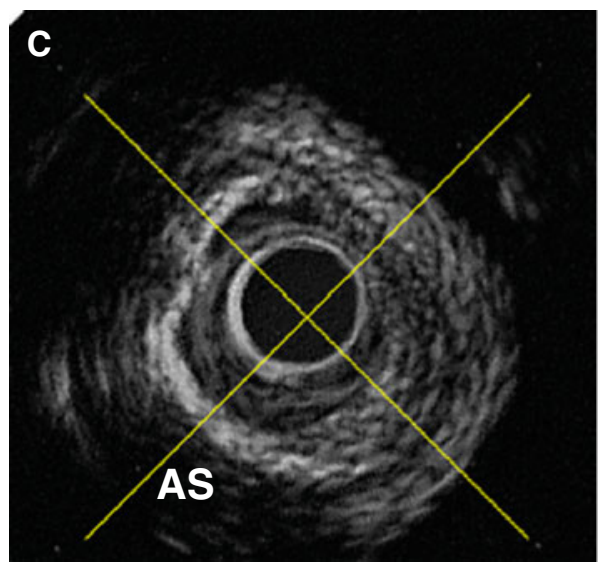

presents the calcium very well between 6 and almost 12 o'clock. Also within the IVUS image it can be appreciated that between 6 and 12 o'clock there is a calcium layer present due to the white and bright interface but everything behind it is shadowed and thus IVUS does not contain any information deeper than this interface. When using IVUS as the only source to estimate the plaque composition tissue within these shadowed areas should therefore be classified as unknown tissue

recently introduced bioabsorbable BVS stent (BVS, Abbott Vascular, Santa Clara,CA, USA). This information could help to explore into more detail the overall performance of this new stent platform.

A major limitation of the RF-based methods, as compared to the image-based echogenicity method, is that they do not take into account acoustic shadowed areas. They divide every individual RF-beam into 
small regions of interest and compare the found signal profiles to their databases of known tissue profiles without taking into account what signal is found in front or behind that window (e.g. the larger picture is missing). This results in detection of tissue behind calcified areas, even if on the gray-scale IVUS image there is only shadow visible, thus the RFsignal will most likely contain noise (thick layers of calcium will reflect all acoustic energy back to the transducer causing the typical bright white appearance on gray-scale IVUS images) (Fig. 2). This could lead to two potential biases: (1) an observer related bias as the outer vessel boundaries are shadowed and thus the outer vessel contour must be interpolated by the observer and (2) a software related bias as the signal processing tools are not taking into account these shadowed areas and will assign the pixels in these regions to any of the tissue components within their database based on the "noise" of the ultrasound waves related to that area. In contrast, the imagebased method of echogenicity at first examines every cross-sectional IVUS image if it contains acoustic shadowed areas and classifies tissue within these areas as unknown preventing possible software related deviations.

Although IVUS is proven to be safe and is a wellestablished method widely available, there are other promising imaging methods, such as optical coherence tomography, but for the time being they are not capable identifying quantitatively tissue components comparable to the described methods in this paper.

In-depth post-hoc analysis of IVUS data can be applied to quantify coronary plaque composition and possible changes of this composition over time which can be aplied to evaluate new therapeutic treatment methods. However, there is still a large scientific debate how these different analysis methods exactly relate to each other and to that of the golden standard of histopathology. If these methods are also capable of identifying possible vulnerable segments is still under investigation.

Open Access This article is distributed under the terms of the Creative Commons Attribution Noncommercial License which permits any noncommercial use, distribution, and reproduction in any medium, provided the original author(s) and source are credited.

Conflict of interest None.

\section{References}

1. Roelandt JR, Serruys PW, Bom N, Gussenhoven WG, Lancee CT, ten Hoff H (1989) Intravascular real-time, twodimensional echocardiography. Int $\mathrm{J}$ Card Imaging 4: 63-67

2. Mintz GS, Nissen, SE, Anderson, WD, Bailey, SR, Erbel R, Fitzgerald PJ, Pinto FJ, Rosenfield K, Siegel RJ, Tuzcu EM, Yock PG (2001) American College of Cardiology Clinical Expert Consensus Document on Standards for Acquisition, Measurement and Reporting of Intravascular Ultrasound Studies (IVUS). A report of the American College of Cardiology Task Force on Clinical Expert Consensus Documents. J Am Coll Cardiol 37:1478-1492

3. Nissen SE, Yock P (2001) Intravascular ultrasound: novel pathophysiological insights and current clinical applications. Circulation 103:604-616

4. Serruys PW, Ormiston JA, Onuma Y et al (2009) A bioabsorbable everolimus-eluting coronary stent system (ABSORB): 2-year outcomes and results from multiple imaging methods. Lancet 373:897-910

5. Serruys PW, Garcia-Garcia HM, Buszman P et al (2008) Effects of the direct lipoprotein-associated phospholipase $\mathrm{A}(2)$ inhibitor darapladib on human coronary atherosclerotic plaque. Circulation 118:1172-1182

6. Nissen SE, Nicholls SJ, Sipahi I et al (2006) Effect of very high-intensity statin therapy on regression of coronary atherosclerosis: the ASTEROID trial. JAMA 295: 1556-1565

7. Nissen SE, Nicholls SJ, Sipahi I et al (2006) Effect of very high-intensity statin therapy on regression of coronary atherosclerosis: the ASTEROID trial. JAMA 295: 1556-1565

8. Nissen SE, Nicholls SJ, Wolski K et al (2008) Comparison of pioglitazone vs glimepiride on progression of coronary atherosclerosis in patients with type 2 diabetes: the PERISCOPE randomized controlled trial. JAMA 299: $1561-1573$

9. Nissen SE, Nicholls SJ, Wolski K et al (2008) Effect of rimonabant on progression of atherosclerosis in patients with abdominal obesity and coronary artery disease: the STRADIVARIUS randomized controlled trial. JAMA 299:1547-1560

10. Nissen SE, Tardif JC, Nicholls SJ et al (2007) Effect of torcetrapib on the progression of coronary atherosclerosis. N Engl J Med 356:1304-1316

11. Nissen SE, Tsunoda T, Tuzcu EM et al (2003) Effect of recombinant ApoA-I Milano on coronary atherosclerosis in patients with acute coronary syndromes: a randomized controlled trial. JAMA 290:2292-2300

12. Nissen SE, Tuzcu EM, Brewer HB et al (2006) Effect of ACAT inhibition on the progression of coronary atherosclerosis. N Engl J Med 354:1253-1263

13. Nissen SE, Tuzcu EM, Libby P et al (2004) Effect of antihypertensive agents on cardiovascular events in patients with coronary disease and normal blood pressure: the CAMELOT study: a randomized controlled trial. JAMA 292:2217-2225 
14. Nissen SE, Tuzcu EM, Schoenhagen P et al (2004) Effect of intensive compared with moderate lipid-lowering therapy on progression of coronary atherosclerosis: a randomized controlled trial. JAMA 291:1071-1080

15. Nair A, Kuban BD, Tuzcu EM, Schoenhagen P, Nissen SE, Vince DG (2002) Coronary plaque classification with intravascular ultrasound radiofrequency data analysis. Circulation 106:2200-2206

16. Nair A, Margolis MP, Kuban BD, Vince DG (2007) Automated coronary plaque characterisation with intravascular ultrasound backscatter: ex vivo validation. EuroIntervention 3:113-120

17. Sathyanarayana S, Carlier S, Li W, Thomas L (2009) Characterisation of atherosclerotic plaque by spectral similarity of radiofrequency intravascular ultrasound signals. EuroIntervention 5:133-139

18. Okubo M, Kawasaki M, Ishihara Y et al (2008) Development of integrated backscatter intravascular ultrasound for tissue characterization of coronary plaques. Ultrasound Med Biol 34:655-663

19. Bruining N, Verheye S, Knaapen M et al (2007) Threedimensional and quantitative analysis of atherosclerotic plaque composition by automated differential echogenicity. Catheter Cardiovasc Interv 70:968-978

20. Bartorelli AL, Potkin BN, Almagor Y, Keren G, Roberts WC, Leon MB (1990) Plaque characterization of atherosclerotic coronary arteries by intravascular ultrasound. Echocardiography 7:389-395

21. Garcia-Garcia HM, Mintz GS, Lerman A et al (2009) Tissue characterisation using intravascular radiofrequency data analysis: recommendations for acquisition, analysis, interpretation and reporting. EuroIntervention 5:177-189

22. Kawasaki M, Takatsu H, Noda T et al (2002) In vivo quantitative tissue characterization of human coronary arterial plaques by use of integrated backscatter intravascular ultrasound and comparison with angioscopic findings. Circulation 105:2487-2492

23. Kawasaki M, Bouma BE, Bressner J et al (2006) Diagnostic accuracy of optical coherence tomography and integrated backscatter intravascular ultrasound images for tissue characterization of human coronary plaques. J Am Coll Cardiol 48:81-88

24. Palmer ND, Northridge D, Lessells A, McDicken WN, Fox KA (1999) In vitro analysis of coronary atheromatous lesions by intravascular ultrasound; reproducibility and histological correlation of lesion morphology. Eur Heart $\mathbf{J}$ 20:1701-1706

25. Prati F, Arbustini E, Labellarte A et al (2001) Correlation between high frequency intravascular ultrasound and histomorphology in human coronary arteries. Heart $85: 567-570$

26. Nasu K, Tsuchikane E, Katoh O et al (2006) Accuracy of in vivo coronary plaque morphology assessment: a validation study of in vivo virtual histology compared with in vitro histopathology. J Am Coll Cardiol 47:2405-2412

27. Granada JF, Wallace-Bradley D, Win HK et al (2007) In vivo plaque characterization using intravascular ultrasound-virtual histology in a porcine model of complex coronary lesions. Arterioscler Thromb Vasc Biol 27:387-393

28. Van Herck J, De Meyer G, Ennekens G, Van Herck P, Herman A, Vrints C (2009) Validation of in vivo plaque characterisation by virtual histology in a rabbit model of atherosclerosis. EuroIntervention 5:149-156

29. Thim T, Hagensen MK, Wallace-Bradley D, Granada JF, Kaluza GL, Drouet L, Paaske WP, Bøtker HE, Falk E (2010) Unreliable assessment of necrotic core by VHTM IVUS in porcine coronary artery disease. Circ Cardiovasc Imaging 3(4):384-391 